\title{
A White Light Megaflare on the dM4.5e Star YZ $\mathrm{CMi}^{1}$
}

\author{
Adam F. Kowalski², Suzanne L. Hawley², Jon A. Holtzman ${ }^{3}$, John P. Wisniewski ${ }^{2,4}$, Eric J. \\ Hilton $^{2}$
}

\begin{abstract}
On UT 2009 January 16, we observed a white light megaflare on the dM4.5e star YZ CMi as part of a long-term spectroscopic flare-monitoring campaign to constrain the spectral shape of optical flare continuum emission. Simultaneous

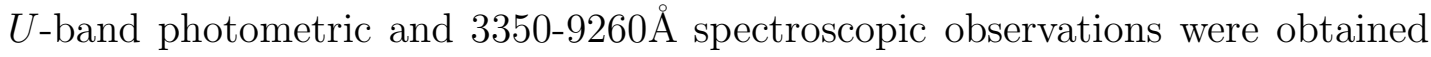
during 1.3 hours of the flare decay. The event persisted for more than 7 hours and at flare peak, the $U$-band flux was almost 6 magnitudes brighter than in the quiescent state. The properties of this flare mark it as one of the most energetic and longest-lasting white light flares ever to be observed on an isolated low-mass star. We present the $U$-band flare energetics and a flare continuum analysis. For the first time, we show convincingly with spectra that the shape of the blue continuum from $3350 \AA$ to $4800 \AA$ can be represented as a sum of two components: a Balmer continuum as predicted by the Allred et al radiative hydrodynamic flare models and a $T \sim 10,000 \mathrm{~K}$ blackbody emission component as suggested by many previous studies of the broadband colors and spectral distributions of flares. The areal coverage of the Balmer continuum and blackbody emission regions vary during the flare decay, with the Balmer continuum emitting region always being significantly ( $\sim 3-16$ times) larger. These data will provide critical constraints for understanding the physics underlying the mysterious blue continuum radiation in stellar flares.
\end{abstract}

Subject headings: stars: flare — stars: late-type — stars: atmospheres — stars: individual (YZ CMi)

\footnotetext{
${ }^{1}$ Based on observations obtained with the Apache Point Observatory 3.5-meter telescope, which is owned and operated by the Astrophysical Research Consortium.

${ }^{2}$ Astronomy Department, University of Washington, Box 351580, Seattle, WA 98195 email: kowalski@astro.washington.edu

${ }^{3}$ Department of Astronomy, New Mexico State University, Box 30001, Las Cruces, NM 88003

${ }^{4}$ NSF Astronomy \& Astrophysics Postdoctoral Fellow
} 


\section{Introduction}

Due to their strong and persistent surface magnetic fields, active $\mathrm{M}$ dwarfs can produce frequent flares, sometimes lasting for many hours and reaching luminosities that approach a significant fraction of the star's bolometric luminosity (Kunkel 1969; Moffett 1974; Bond 1976; Hawley \& Pettersen 1991; Favata et al. 2000; Robinson et al. 2005; Osten et al. 2007; Kowalski et al. 2009). During both solar and stellar flares, emission is seen as line and continuum radiation from X-ray to radio wavelengths, with a prominent mode of radiative energy release occurring in the blue and near-ultraviolet (NUV) continuum, which is commonly referred to as the white light continuum. Unfortunately, the spectral components which comprise the white light continuum are not well-constrained, and therefore the physics of the underlying flare mechanism that gives rise to such a conspicuous signature remains a mystery.

Significant effort has gone into characterizing the white light continuum in stellar flares, beginning with a single component (hydrogen recombination) model (Kunkel 1969, 1970). A two-component spectral model consisting of hydrogen recombination and an impulsively heated photosphere was first proposed by Kunkel (1970) as a possible explanation of the spread in broadband flare colors. Time-resolved multi-channel photometry (Mochnacki \& Zirin 1980) and spectrometry (Kahler et al. 1982) were obtained longward of $4200 \AA$ for flares on YZ CMi and UV Ceti; at peak, these flare spectra were fit with a Planck function of $T=$ $7400-9500 \mathrm{~K}$, thereby showing evidence of a hot optically thick component present during the

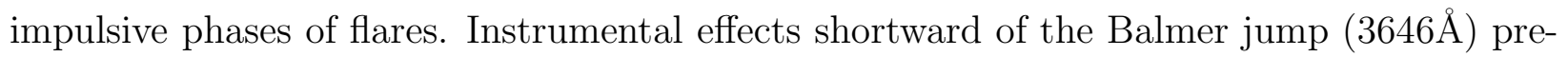
vented a precise characterization of the Balmer continuum in the data of Mochnacki \& Zirin $(1980)$.

Higher resolution spectra in the blue/NUV were obtained during large flares on AD Leo

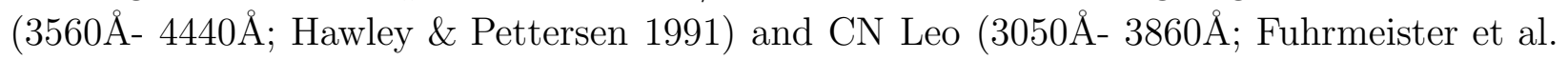
2008) showing a nearly continuous rise into the NUV with a $T \sim 8400-11$, 300K blackbody; surprisingly no discontinuity at the Balmer jump was seen in either of these events (see also Eason et al. 1992; García-Alvarez et al. 2002). The white light continuum has therefore been attributed to a single dominant blackbody component with $T \sim 9000-10,000 \mathrm{~K}$ (e.g., Hawley et al. 2003). In contrast, flare models predict spectra that are dominated by hydrogen bound-free continua, which provide a poor match to the observations (Hawley \& Fisher 1992; Allred et al. 2006, hereafter A06).

A multi-component model was reintroduced by Zhilyaev et al. (2007), who used highcadence $U B V R I$ photometric observations of the dM3.5e star EV Lac to suggest that the white light emission consists primarily of blackbody radiation at flare peak and hydrogen continuum during the flare decay. However, significant ambiguity results when using broad- 
band photometry to characterize the white light continuum. A06 showed that a flare model spectrum that included a large Balmer jump, when convolved with broadband $U V+U B V R$ filters, actually resembles the shape of a $T \sim 9,000 \mathrm{~K}$ blackbody. To unambiguously identify the spectral components present during stellar flares, we have therefore begun to compile a large catalog of time-resolved optical/NUV flare spectra on several nearby flare stars. In this Letter, we present time-resolved spectra obtained during a large flare on the dM4.5e star YZ CMi and use results from the recent radiative hydrodynamic (RHD) flare models of A06 to show that both Balmer continuum and hot blackbody components are present in the white light continuum emission. An extensive paper describing our flare catalog, including discussion of the flare emission lines and comparison with models will be forthcoming.

\section{Observations}

\subsection{NMSU 1-m Photometry}

Photometric observations were obtained in the Johnson $U$ band on UT 2009 January 16 with the New Mexico State University 1-m Telescope at the Apache Point Observatory (APO). The 1-m is operated robotically and is open for observations when the ARC 3.5-m Telescope, located at the same site, is open (Holtzman et al.2010). The dM4.5e star YZ CMi was observed for nearly 8 hours with an exposure time of 10s; with readout the photometric cadence ranged between 20 - 27s. We performed differential photometry on YZ CMi using the comparison star HD 62525. The night was clear and photometric based on the data from nearby comparison stars. The $U$-band light curve of YZ CMi is shown in Figure 1a.

\subsection{APO 3.5-m Spectroscopy}

We obtained NUV/optical spectroscopy for 1.3 hours during the decay phase of the flare, denoted by vertical lines in Figure 1a, using the Dual Imaging Spectrograph (DIS) with the ARC 3.5-m Telescope at APO. We used the B400+R300 gratings, providing wavelength coverage from $3350 \AA-9260 \AA$. The observations were taken through a 1.5 " slit which yielded

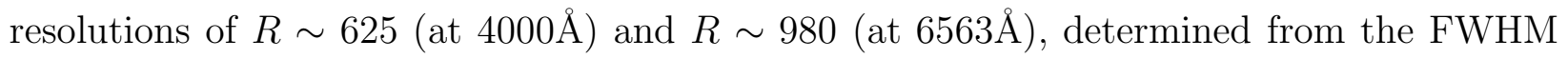
of the arc lamp lines. The slit was oriented at the parallactic angle in order to compensate for atmospheric differential refraction (see Filippenko 1982). We observed YZ CMi with exposure times of $10 \mathrm{~s}$, and with readout the total cadence was $\sim 28 \mathrm{~s}$. In total, 163 flare spectra were obtained simultaneously in both the blue and red bandpasses. The data were 
reduced using standard IRAF1 procedures, wavelength calibrated using HeNeArHg lamp spectra, and flux-calibrated by comparing observations of the spectrophotometric standard star Feige 34 to the accepted fluxes of Oke (1990). To correct for variable grey slit loss (e.g., due to guiding errors), we also scaled each spectrum during the flare using the $U$-band light curve. A spectrum of YZ CMi in its quiescent (non-flaring) state was obtained with the same instrumental setting on UT 2008 November 24 and was used for comparison (see §3.2).2

\section{Analysis}

\subsection{U-Band Flare Energetics}

This flare event is remarkable in its total energy, peak luminosity, total duration, and emission morphology. A small precursor, sometimes seen prior to large flares (Moffett 1974), began at 04:14:54UTC which increased the $U$-band flux by a factor of $\sim 2$, and represents the onset of the flare event. At 04:32:00UTC, there was a $~ 10 \mathrm{~min}$ impulsive rise to flare peak, where the $U$-band flux was a factor of $\sim 200$ times the quiescent value. The decay phase included many smaller events, and the $U$-band flux was still elevated by over a magnitude above quiescence at the end of the night. In $\S 4$, we discuss the extremely long duration of this event.

The total $U$-band flare energy is used to quantify the energy release in continuum radiation during stellar flares (Moffett 1974). We integrate under the light curve to obtain an equivalent duration (Gershberg 1972) of $4.25 \times 10^{5} \mathrm{~s}$, which is the amount of time that the star would spend emitting at the pre-flare level to produce the same total amount of energy as the flare. Multiplying the equivalent duration by the non-flaring $U$-band luminosity of $4.00 \times 10^{28} \mathrm{ergs} \mathrm{s}^{-1}$ (Moffett 1974) gives $E_{U, \text { flare }}>1.7 \times 10^{34} \mathrm{ergs}$ (this is a lower limit since flare emission is still seen at the time the light curve ends). At peak, the flare was emitting with a $U$-band luminosity of $8.3 \times 10^{30} \mathrm{ergs} \mathrm{s}^{-1}$, or $\sim 37 \%$ the stellar bolometric luminosity $\left(\mathrm{M}_{B o l}=10.25\right.$, Reid \& Hawley 2005). This flare was $\sim 100$ times more energetic and persisted for more than 10 times as long as the largest $U$-band flare event observed on this star during 55 hours of monitoring from the large statistical flare study of Moffett (1974)

\footnotetext{
${ }^{1}$ IRAF is distributed by the National Optical Astronomy Observatories, which are operated by the Association of Universities for Research in Astronomy, Inc., under cooperative agreement with the National Science Foundation

${ }^{2}$ The quiescent spectrum was convolved with the Johnson $B$ and $V$ bands (using filter curves from Maíz Apellániz (2006)) and scaled to match the flux from the literature $(V=11.2, B=12.8$; Reid \& Hawley 2005)
} 
and Lacy et al. (1976). Although it is unknown if the power law fit to the flare-frequency distribution of Lacy et al. (1976) holds at high flare energies, an extrapolation predicts that flares with $U$-band energies $>1.7 \times 10^{34}$ ergs occur at a rate of $\sim$ once per month.

\subsection{The Flare Continuum}

The spectral data were obtained while the $U$-band emission was still elevated 15-37 times (3-4 mag) over quiescence. Complex morphology is seen in the $U$-band light curve during this time period (see Figure 1a), allowing us to study the flare continuum variations during several smaller impulsive events. Figure 1 1 b shows a spectrum $(t=76.6 \mathrm{~min}$ after the flare start) from $3350 \AA-5500 \AA$ near the beginning of our spectroscopic observations during the $U$-band decay from a large secondary emission peak. A quiescent spectrum of YZ CMi (see §2.2) is also shown in Figure 1b and has been subtracted from the flare spectrum to give a "flare-only" spectrum. Our spectra have the highest time resolution with high signal to noise in the blue/NUV (S/N > 50 at $3600 \AA$ ) ever obtained during a large stellar flare. They are also unique in having simultaneous high quality photometric information so that we may relate the spectral evolution to the light curve morphology.

We have for the first time convincingly identified two distinct continuum components that simultaneously contribute to the optical/NUV flux in flare spectra. The first component dominates the spectrum from $4000-4800 \AA$ and exhibits the rising trend of a $T \sim 10,000 \mathrm{~K}$ blackbody, which is plotted on the spectrum in Figure $1 \mathrm{~b}$ using a filling factor, $\mathrm{X}_{B B}$, of $0.22 \%$ of the projected stellar disk area. We follow Hawley et al. (2003) to constrain the filling factor of blackbody emission as a function of time using the equation:

$$
F_{f l, \lambda}(t)=X_{B B}(t) \frac{R_{\star}^{2}}{d^{2}} \pi B_{\lambda}\left(T_{f l}\right)
$$

where $F_{f l, \lambda}$ is the flare flux observed at Earth in the continuum windows from 3995-4020 $\AA$, $4140-4210 \AA, 4425-4450 \AA$, and $4600-4800 \AA ; R_{\star}=0.3 \times R_{\text {Sun }}, d=5.97$ pc (Reid \& Hawley 2005) and $T_{f l}=10,000 \mathrm{~K}$.

From Figure 1b, it is clear that the blackbody fit does not explain all of the continuum

emission at wavelengths shorter than $\sim 3800 \AA$. Starting near H10 (3799 $)$, there is a rise to $\mathrm{H} 15+\mathrm{H} 16$, the last two apparent Balmer lines in emission. Blueward of $\sim 3700 \AA$, the flare spectrum becomes flat into the NUV, and there is excess flux visible above the $10,000 \mathrm{~K}$ blackbody at these wavelengths.

A06 presented RHD flare simulations for a medium (F10) and a strong (F11) impulsive 
flux of nonthermal electrons into an $\mathrm{M}$ dwarf atmosphere. The A06 models predict strong Balmer continuum radiation with a noticeable Balmer jump at $3646 \AA$, together with a photosphere that is warmed by $\sim 1200 \mathrm{~K}$ to a temperature of $4600 \mathrm{~K}$. We isolated the Balmer continuum component of the A06 F11 model spectrum taken at the last time-step of their simulation $(t=15.9 \mathrm{~s})$, and fit it to the observed flare spectra. Figure 1 1 shows the flare spectrum from Figure 1b with the 10,000K blackbody component subtracted and the model Balmer continuum component scaled to the excess emission in the $3600 \AA<\lambda<3646 \AA$ region. The shape of the observed excess emission is well-described by the F11 flare model prediction (shown in red). Although there is no abrupt discontinuity indicative of a Balmer jump in our observed spectrum, we posit that blending (possibly due to Stark broadening; Donati-Falchi et al. 1985) of the higher order Balmer lines in the $3646 \AA<\lambda<3800 \AA$ region effectively hides the sharp discontinuity that is seen in the model spectrum.

\subsection{Relative Filling Factors}

The filling factor for the F11 model spectrum is calculated by scaling the model flux in the $3600-3646 \AA$ region $\left(F_{\lambda, \text { Model }}=1.14 \times 10^{7} \mathrm{ergs} \mathrm{cm}^{-2} \mathrm{~s}^{-1} \AA^{-1}\right)$ to the flux excess observed at Earth after subtracting the $10,000 \mathrm{~K}$ blackbody. The contribution function for the Balmer continuum in the F11 model shows that the emission arises from a relatively thin layer in the atmosphere (J. Allred, private communication); it is therefore reasonable to approximate changes in the filling factor to changes in the emitting area of the Balmer continuum.

In the upper panel of Figure 1d (black points), we show the ratio of the filling factors for a $10,000 \mathrm{~K}$ blackbody and the model Balmer continuum component. The error bars are obtained by using 9000 and 11,000K temperatures for the blackbody. The ratio for the sample spectrum in Figure 1b is $X_{B B} / X_{\text {Model }}=0.10 \pm_{0.02}^{0.04}$. The filling factor ratio indicates that the two emission components exhibit significantly different time evolution, with the blackbody source emitting from a region $\sim 3-16$ times smaller than the source of the Balmer continuum.

The inferred area filling factor $\left(X_{B B}\right)$ of the blackbody component is shown separately (red points) and exhibits very similar morphology to the $U$-band light curve (reproduced in the lower panel). Although we expect the total continuum flux to follow the $U$-band since we scaled the spectra to achieve absolute flux calibration ( $(3.2)$, it is surprising that only the blackbody component follows the $U$ band 3 It appears that the deviations from the general flare decay seen in the $U$ band primarily arise from the changing area of the

\footnotetext{
${ }^{3}$ These trends are also visible in the data before the flux calibration scaling was applied.
} 
blackbody emission region. When the deviations are strongest near the peaks at $t \sim 95$ min and $t \sim 130 \mathrm{~min}$, the spectra are increasingly dominated by the blackbody component. This is consistent with the flare-peak spectra from Kahler et al. (1982), Hawley \& Pettersen (1991), and Fuhrmeister et al. (2008) and the photometry of Zhilyaev et al. (2007). We also note preliminary evidence of an apparent increase in the best-fit blackbody temperature to $T \sim 13,000 \mathrm{~K}$ at the peak near $t \sim 130 \mathrm{~min}$.

The derived filling factor ratios should be regarded as illustrative since our Balmer continuum spectrum from the F11 model of A06 assumes that the nonthermal electron flux is constant at $10^{11} \mathrm{ergs} \mathrm{cm}^{-2} \mathrm{~s}^{-1}$. Also, we use the model flare spectrum from only one time-step in the RHD simulation. It is likely that the nonthermal electron flux varied both with time and over the geometry of the flaring region. Any further assessment is beyond the scope of this Letter, but we note that the less energetic A06 F10 flare spectrum at $t=230.0 \mathrm{~s}$ gives nearly 10 times larger filling factors for the Balmer continuum.

\subsection{Balmer Line and Continuum Radiation}

The time-evolution of the Balmer continuum closely follows the hydrogen Balmer lines. We show the variation in the Balmer continuum (using the integrated flux in a $30 \AA$ window centered at $3615 \AA$ ) compared to the $\mathrm{H} \gamma$ line flux and the $U$ band in the lower panel of Figure 1d. The fluxes are normalized to the last spectral observation we obtained. The Balmer continuum shows a slow decay throughout our observations, as is typical of the Balmer line radiation (Hawley \& Pettersen 1991). This overall decay is probably extended emission from the previous, much more luminous, impulsive events seen in the $U$-band light curve. The larger variations in the Balmer continuum compared to the $\mathrm{H} \gamma$ emission may be partially due to small errors in isolating the Balmer continuum by subtracting the underlying blackbody continuum (the $\mathrm{H} \gamma$ flux is well measured as it has a local continuum). As noted above in the filling factor discussion and evident by comparing the upper and lower panels of Figure $1 \mathrm{~d}$, the Balmer line and continuum radiation exhibit quite different time evolution compared to the blackbody emission component. We propose two possible explanations:

(a) The hydrogen-emitting regions exhibit a delayed and more gradual response to each successive impulsive (blackbody) event. In this scenario, the gradual increase in hydrogen Balmer emission around $t=122$ and $t=137 \mathrm{~min}$ are associated with the impulsive events in blackbody emission (upper panel) at $t=117 \mathrm{~min}$ and $t=127 \mathrm{~min}$, respectively. Also, Balmer emission around $t=80 \mathrm{~min}$ and $t=105 \mathrm{~min}$ is elevated yet only slowly decreasing, and could be gradually declining emission from the large $U$-band peaks at $t \sim 60$ min and $t \sim 95 \mathrm{~min}$, respectively. 
(b) The time evolution of the blackbody emission and the Balmer emission may be anticorrelated. If true, this may provide an important constraint on the origin of the blackbody emission. The possible anti-correlation is most apparent during the $U$-band rise starting at $t=124 \mathrm{~min}$. In $\sim 5$ min the Balmer continuum decreases by $\sim 40 \%$ while the blackbody filling factor almost doubles. Hydrogen continuum dimming is predicted by flare models (Abbett \& Hawley 1999; Allred et al. 2005), but only for very short durations $(0.1 \mathrm{~s})$ at the onset of a flare.

More detailed models will be required to understand these complex variations, and in particular, the seemingly anti-correlated trends between the blackbody and Balmer continuum and lines during the time intervals when the blackbody filling factor is increasing in Figure 1].

\section{Summary and Speculation}

We observed a white light megaflare on the dM4.5e star YZ CMi in the $U$ band and with simultaneous optical/NUV spectroscopy. The $U$-band energetics and light curve morphology qualify this flare as an extraordinary and rare event, similar to the $\sim 6.6 \times 10^{34} \mathrm{erg}$ flare on YZ CMi seen by Andrews et al. (1969), Lovell (1969), and Kunkel (1969). Following a solar analogy, we speculate that the flaring region on YZ CMi was a complex arcade of sequentially reconnecting magnetic loops. Each reconnecting loop accelerated a beam of nonthermal electrons that impacted the lower atmosphere, producing the observed blue/NUV line and continuum emission. The sum of a large number of individual emitting regions may have enabled this flare to persist for such an unusually long time.

Using high time resolution, high signal-to-noise spectra, we have shown that the blue/NUV flare continuum radiation can be explained as a sum of a $T \sim 10,000 \mathrm{~K}$ blackbody component and a Balmer continuum component, with only the blackbody emission present from

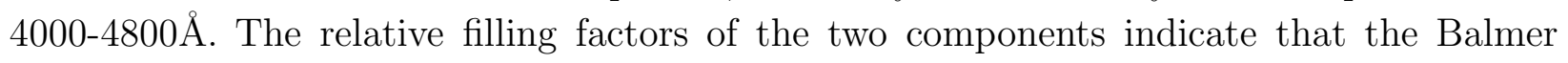
continuum comes from a larger region, plausibly originating from the flaring loops at chromospheric heights, where the Balmer lines originate. This is consistent with the height of the Balmer continuum emission derived from solar flare data (Hudson et al. 2010), and also with the height of formation predicted by the RHD model of A06.

We found that the blackbody emission arises from a region $\sim 3-16$ times smaller in area than the Balmer continuum emission region. This blackbody emission may possibly originate in concentrated magnetic footpoint regions in the lower atmosphere, similar to the localized areas that emit in white light during solar flares (Metcalf et al. 2003; Fletcher et al. 
2007; Isobe et al. 2007; Jess et al. 2008), which are often spatially and temporally coincident with impulsive heating by nonthermal electrons inferred from hard X-ray observations (e.g., Rust \& Hegwer 1975; Hudson et al. 1992; Neidig \& Kane 1993; Fletcher et al. 2007). In accordance with this scenario, a large complex of photospheric hot spots may have been created during the first impulsive events in the YZ CMi $U$-band light curve. As the nonthermal electron beams weakened (in energy and/or in spatial extent) so did the areal coverage of these hot spots. This gradual decay extends into the time covered by our spectral observations and is seen in the overall decline of the blackbody areal coverage in the upper panel of Figure 11. During our spectral observations, a few new hot spots may have been created at the footpoints of newly reconnected loops, causing the transient increases seen in the $U$-band and in the effective area of the blackbody emitting region.

We see strong evidence through all of our observations for a blackbody continuum emission component with an approximate temperature of $10,000 \mathrm{~K}$. The persistence of the hot blackbody emission indicates that a continual source of particle acceleration and plasma heating likely still exists during the decay phase of the flare. Yet, the A06 models, which employ a nonthermal electron beam as is seen on the Sun, predict the photosphere of an M dwarf to be heated by at most $\sim 1200 \mathrm{~K}$ during a large flare. The physical mechanism which generates the strong blackbody emission (presumably from a more strongly heated photosphere) therefore remains unknown. The possible anti-correlation between the Balmer continuum and blackbody emission components may provide an important clue to the nature of the heating mechanism that is responsible for the blackbody emission.

\section{Acknowledgments}

AFK, SLH, and EJH acknowledge support from NSF grant AST 08-07205. JPW aknowledges support from NSF AAPF AST 08-02230. We would like to thank B. Williams, J. Allred, and E. Böhm-Vitense for their helpful conversations.

\section{REFERENCES}

Abbett, W. P., \& Hawley, S. L. 1999, ApJ, 521, 906

Allred, J. C., Hawley, S. L., Abbett, W. P., \& Carlsson, M. 2005, ApJ, 630, 573

Allred, J. C., Hawley, S. L., Abbett, W. P., \& Carlsson, M. 2006, ApJ, 644, 484 
Andrews, A. D., Corvan, P., Hardy, B., Johnston, P., Johnston, W., \& Perrott, J. 1969, Information Bulletin on Variable Stars, 325, 1

Bond, H. E. 1976, Information Bulletin on Variable Stars, 1160, 1

Donati-Falchi, A., Falciani, R., \& Smaldone, L. A. 1985, A\&A, 152, 165

Eason, E. L. E., Giampapa, M. S., Radick, R. R., Worden, S. P., \& Hege, E. K. 1992, AJ, 104,1161

Favata, F., Reale, F., Micela, G., Sciortino, S., Maggio, A., \& Matsumoto, H. 2000, A\&A, 353,987

Filippenko, A. V. 1982, PASP, 94, 715

Fletcher, L., Hannah, I. G., Hudson, H. S., \& Metcalf, T. R. 2007, ApJ, 656, 1187

Fuhrmeister, B., Liefke, C., Schmitt, J. H. M. M., \& Reiners, A. 2008, A\&A, 487, 293

García-Alvarez, D., Jevremović, D., Doyle, J. G., \& Butler, C. J. 2002, A\&A, 383, 548

Gershberg, R. E. 1972, Ap\&SS, 19, 75

Hawley, S. L., et al. 2003, ApJ, 597, 535

Hawley, S. L., \& Fisher, G. H. 1992, ApJS, 78, 565

Hawley, S. L., \& Pettersen, B. R. 1991, ApJ, 378, 725

Holtzman, J. A., Harrison, T. E., \& Coughlin, J. L. 2010, in press

Hudson, H., Fletcher, L., \& Krucker, S. 2010, ArXiv e-prints

Hudson, H. S., Acton, L. W., Hirayama, T., \& Uchida, Y. 1992, PASJ, 44, L77

Isobe, H., et al. 2007, PASJ, 59, 807

Jess, D. B., Mathioudakis, M., Crockett, P. J., \& Keenan, F. P. 2008, ApJ, 688, L119

Kahler, S., et al. 1982, ApJ, 252, 239

Kowalski, A. F., Hawley, S. L., Hilton, E. J., Becker, A. C., West, A. A., Bochanski, J. J., \& Sesar, B. 2009, AJ, 138, 633

Kunkel, W. E. 1969, Nature, 222, 1129 
Kunkel, W. E. 1970, ApJ, 161, 503

Lacy, C. H., Moffett, T. J., \& Evans, D. S. 1976, ApJS, 30, 85

Lovell, B. 1969, Nature, 222, 1126

Maíz Apellániz, J. 2006, AJ, 131, 1184

Metcalf, T. R., Alexander, D., Hudson, H. S., \& Longcope, D. W. 2003, ApJ, 595, 483

Mochnacki, S. W., \& Zirin, H. 1980, ApJ, 239, L27

Moffett, T. J. 1974, ApJS, 29, 1

Neidig, D. F., \& Kane, S. R. 1993, Sol. Phys., 143, 201

Oke, J. B. 1990, AJ, 99, 1621

Osten, R. A., Drake, S., Tueller, J., Cummings, J., Perri, M., Moretti, A., \& Covino, S. 2007, ApJ, 654, 1052

2005, New light on dark stars : red dwarfs, low-mass stars, brown dwarfs, ed. Reid, I. N. \& Hawley, S. L.

Robinson, R. D., et al. 2005, ApJ, 633, 447

Rust, D. M., \& Hegwer, F. 1975, Sol. Phys., 40, 141

Zhilyaev, B. E., et al. 2007, A\&A, 465, 235 

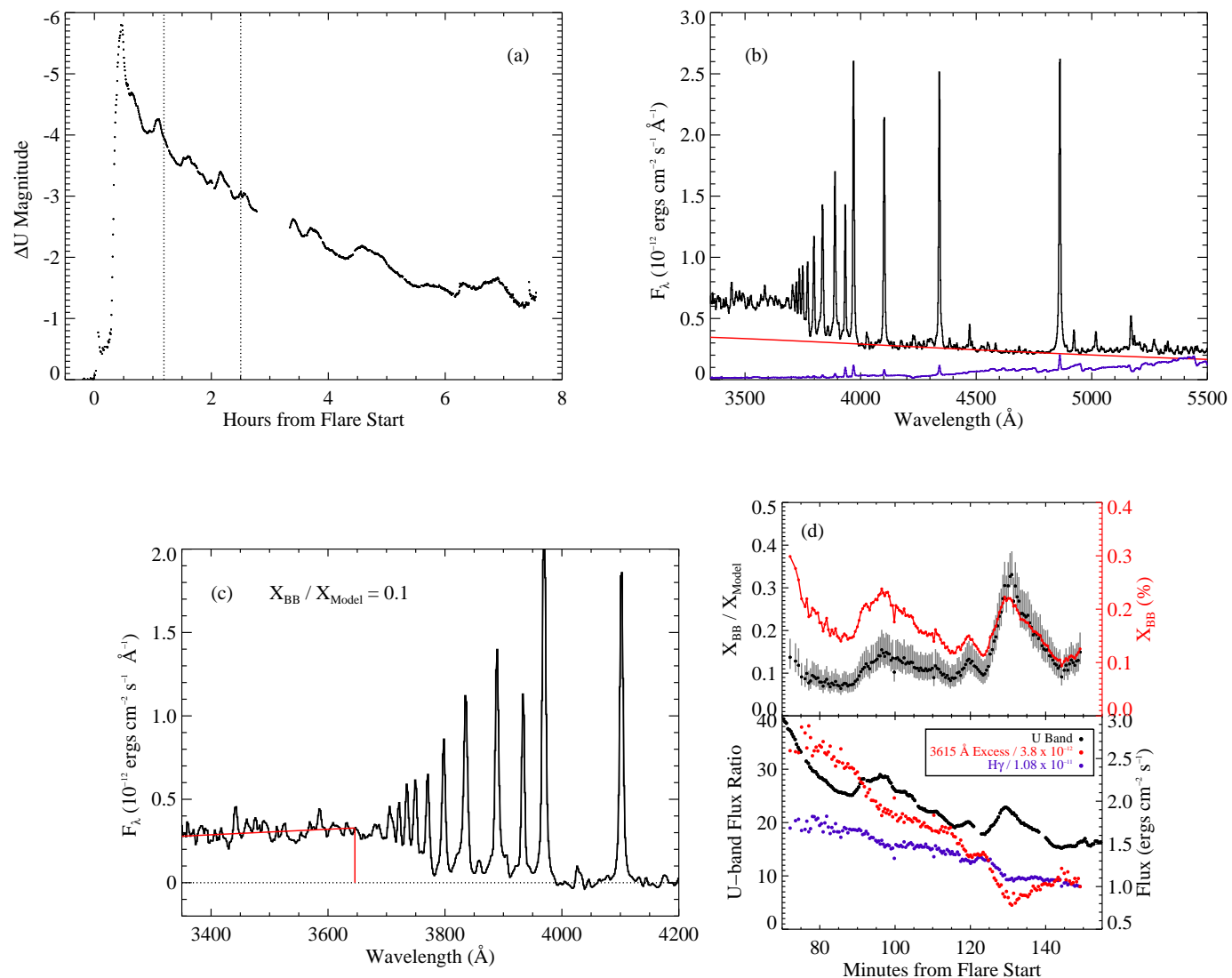

Fig. 1.- (a) The flare $U$-band light curve with vertical lines indicating the period of spectroscopic observations. (b) A flare spectrum at $t=76.6 \mathrm{~min}$ after flare start. The quiescent spectrum is shown in purple for comparison. The continuum from $4000-4800 \AA$ was fit with a $T=10,000 \mathrm{~K}$ blackbody and a filling factor of $0.22 \%$. There is excess emission above the blackbody blueward of $3800 \AA$. (c) The 10,000K blackbody component has been subtracted from the spectrum in panel (b) and the F11 flare spectrum from A06 fits the excess (Balmer) continuum at wavelengths shorter than $3646 \AA$. (d) (upper panel) The filling factor ratio $\left(X_{B B} / X_{\text {Model }}\right.$, black points) and the inferred area coverage of the blackbody component (red points). (lower panel) The time-evolution of the $U$-band (left axis), and the Balmer continuum and $\mathrm{H} \gamma$ fluxes (right axis). 